\title{
An Augmented Reality Head-Up Display System with a Wide-View Eyebox
}

\author{
Pei-Jung Wu, ${ }^{1}$ Chih-Hao Chuang, ${ }^{2}$ Chien-Yu Chen $\left(\mathbb{D},{ }^{3}\right.$ Jeng-Han Wu, ${ }^{4}$ and Bor-Shyh Lin ${ }^{5}$ \\ ${ }^{1}$ College of Information and Distribution Science, National Taichung University of Science and Technology, \\ Taichung 40401, Taiwan \\ ${ }^{2}$ Graduate Institute of Photonics and Optoelectronics, National Taiwan University, Taipei 106, Taiwan \\ ${ }^{3}$ Graduate Institute of Color and Illumination Technology, National Taiwan University of Science \& Technology, \\ Taipei 10607, Taiwan \\ ${ }^{4}$ Department of Electronic Engineering, National Yunlin University of Science \& Technology, Yunlin 64002, Taiwan \\ ${ }^{5}$ Institute of Imaging and Biomedical Photonics, National Chiao Tung University, Tainan 71150, Taiwan
}

Correspondence should be addressed to Chien-Yu Chen; chencyue@mail.ntust.edu.tw

Received 5 October 2019; Revised 12 May 2020; Accepted 10 June 2020; Published 30 June 2020

Academic Editor: Paramasivam Senthilkumaran

Copyright (C) 2020 Pei-Jung Wu et al. This is an open access article distributed under the Creative Commons Attribution License, which permits unrestricted use, distribution, and reproduction in any medium, provided the original work is properly cited.

\begin{abstract}
This study proposes to apply the combination of two 90-degree prisms and a holographic optical element to an image-dividing element, divide an image into three through the virtual image projection system, and accurately project the images to $160 \mathrm{~cm}$ behind the windshield. In order to distinguish the left image, middle image, and right image at the horizontal direction, the 90 degree prism is first utilized for horizontally deflecting the light, a holographic optical element is then used for presenting the vertical deflection of the image, and finally, the images are horizontally arranged as a real image on the diffuser. An image-dividing element is eventually integrated to the virtual image projection system. Then, the divided images are made as a virtual image which is projected to $160 \mathrm{~cm}$ behind the windshield to combine with the street scene. The horizontal angle of view of an eyebox is increased from $2.51^{\circ}$ to $7.22^{\circ}$, and the size of the eyebox is $31.68 \mathrm{~cm} \times 12.48 \mathrm{~cm}$, allowing a wide-view laser-based head-up display with the large-angle beam-splitting function being successfully designed. It would not affect the field of view on the road, and this system could reduce the space and be arranged easily in a vehicle. Therefore, this proposed system is suitable for the aftermarket.
\end{abstract}

\section{Introduction}

Head-up displays are normally applied to airplanes [1]. Nevertheless, the rapid development of science and technology has such a technique be gradually introduced to automobile display systems. Driving a vehicle is not as complicated as flying an airplane and the surrounding landscape presents few changes. However, when drivers are distracted by watching the information from the dashboard, it is hard for them to keep focusing on the road. Then, the traffic accidents are likely to be enhanced [2]. The development of head-up displays, therefore, could solve such inconvenience for drivers and ensure the safety on streets. In regard to the development of automobile displays, displays have evolved from traditional CRT to liquid-crystal displays
(LCDs) [3], liquid crystal on silicon (LCoS), and digital micromirror devices (DMD) because of the advance of digital technology. The advance of MEMS process in past years further allows the application of laser-based pico projectors [4].

Based on distinct imaging principles, head-up displays could be divided into direct projection HUD and virtual image HUD [5]. The former utilizes optical projection for directly projecting an image to the reflecting coating on the windshield [6] (Figure 1(a)) so as to actually image the traffic information on the reflecting coating in front of the driver. Nonetheless, the transmittance is also reduced to generate visual blind angle. Besides, the imaging position of such a direct projection HUD is close that the visual accommodation is necessary for a driver viewing the image 


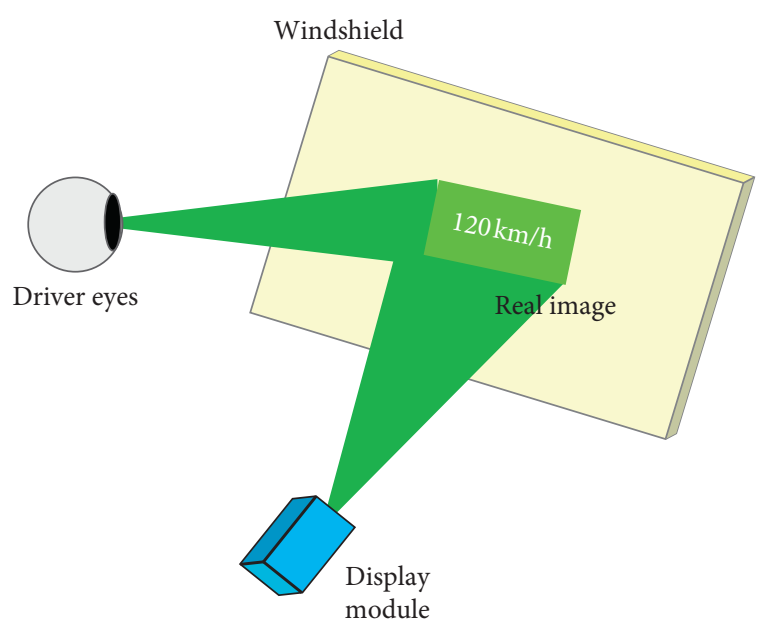

(a)

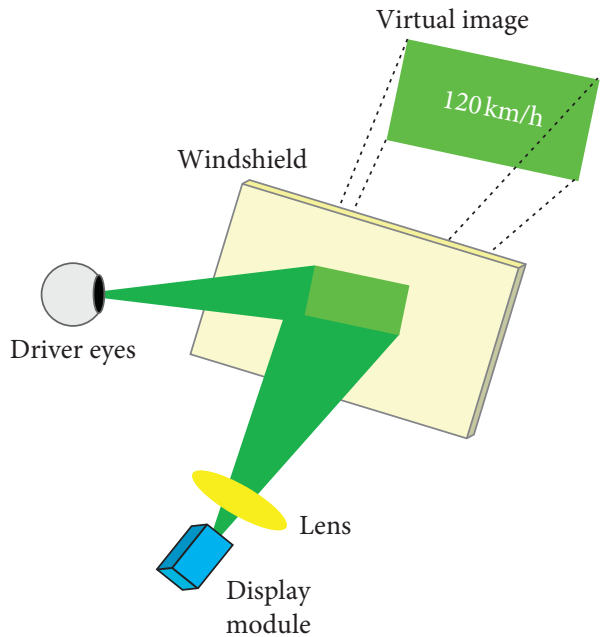

(b)

FIgUre 1: (a) Direct projection HUD; (b) virtual image HUD.

information. The latter merely requires an optical lens to control the imaging distance of a virtual image, without a reflector on the windshield, that the system cost could be reduced [7]. As shown in Figure 1(b), the lens imaging principle is used for controlling the imaging position behind the windshield and combining with the street scene beyond $3 \mathrm{~m}$. In this case, eye-accommodation is not necessary for human eyes that the road safety could be largely enhanced. The construction of this system is huge among the current literature and patents that it is not easily equipped in a vehicle. Besides, the horizontal angle of view of the eyebox is the angle between an image width and human eyes under the premise of not affecting the image quality that a narrow angle could easily result in inconvenient viewing for a driver. According to some previous studies $[8,9]$, the HUD systems based on the principles of total internal reflections and holographic waveguides have some advantages such as decreasing the total volume and increasing the eyebox. However, there are some problems or limitations from the holographic waveguide HUD systems by using many HOEs. First, the dispersion could be caused when the light passing through the HOE. Then, the aberration could be also caused by the flatness of the holographic materials. Third, both the wavelength and the angle of the incident light need to satisfy with the requirements of HOE and the total internal reflection condition of the waveguide. In addition, the volume of the optic system with many HOEs can be decreased but the structure of the HOE system is too difficult to be built for mass production due to the very low tolerance of the HOE system. Besides, it is hard to correct the image after the system completed.

By combining an image-dividing element and the virtual image projection system, a wide-view laser-based head-up display is produced in this study. The high illumination and high power of the laser beam source are used for replacing the LCD image module, and eyebox for automobile head-up displays is designed and expanded. The eyebox of a general virtual image HUD is not wide enough because of the limited image projection size and lens imaging size, and the projected image information is also crowded, resulting in viewing difficulty for drivers. Two 90-degree prisms and a holographic optical element are, therefore, combined to divide images with the characteristics of laser wavelength selectivity and diffraction of the holographic optical element. Not only this system could divide the image projected by the laser-based pico projector into three horizontal images, but also a virtual image projection lens is designed to project such three images to $160 \mathrm{~cm}$ behind the windshield, which is used as a combiner. The volume of this system is not huge. Forevermore, this system can be installed and adjusted for different types of cars easily. It could prevent a driver from looking away from the street and expand the angle of view of the eyebox for a driver.

\section{Materials and Methods}

A laser-based pico projector ( $\mathrm{Pico} \mathrm{P}^{\circledR}$ display) is used for an automobile head-up display outputting the traffic information [10]. Nevertheless, both the vertical and horizontal projection angles of the laser projector are narrow that the image can hardly be enlarged in the limited space through a projection lens. For this reason, an image dividing element is designed to clip and project an original image so as to expand the eyebox of the head-up display, allowing the driver to view more traffic information.

The laser-based pico projector used in this study shows the resolution $848 \times 480$ and the focus range $200 \mathrm{~mm}-$ $2500 \mathrm{~mm}$. In this study, the designed original images are three pieces of vertical traffic information side by side, including speed, direction indication, and warning light, and the intermediate image reveals the size $1 \mathrm{~cm} \times 1 \mathrm{~cm}$. When an image goes through the image dividing element designed in this study, the upper, middle, and lower parts of the original image would be divided into three horizontal images side by side. Such an image dividing element could increase the horizontal field of view and provide more comfortable and secure viewing images for the driver. 
Figure 2 presents the system architecture, covering an image-dividing element and a virtual image projection system. The image-dividing element is designed with the combination of two 90-degree prisms and a holographic optical element. The 90-degree prisms are first used for deflecting the upper-layer image and the lower-layer image to the left and the right, and such two images are deflected to the required positions with the holographic optical element. Furthermore, a diffuser is placed at the intermediate image to make the divided images become an intermediate real image [11]. With the convex lens in the virtual image projection system, the traffic information is virtually projected to $160 \mathrm{~cm}$ behind the windshield. The design and production of the image-dividing element and the holographic optical element are described in the section "ImageDividing Element," and the design and production of the virtual image projection system is demonstrated in the section "Virtual Image Projection System."

2.1. Image-Dividing Element. In this study, an original image can be divided into horizontal images with two 90-degree prisms and the subimages can be vertically moved with a holographic optical element. In Figure 2, a laser-based pico projector is used for projecting an original image, and a prism is used for moving the upper subimage rightward and the lower subimage leftward (Figure 3(a)). The holographic optical element is further used for generating the vertical first-order diffraction of the image to vertically move the subimages on the right top and the left bottom so as to display the three subimages on the same horizontal plane side by side (Figure 3(b)). In addition to assisting two subimages in diffracting to the required positions, the holographic optical element presents the advantages of small size and light weight, and the diffracted image would not be distorted because of refraction [12].

First of all, the relationship between the incident angle and the deviation is utilized for estimating the required parameters of the 90-degree prisms. The laser beam source is used as the incident light in this study. However, the laserbased pico projector has a stable divergence angle. The three divided images might be overlapping partially after the incident light passing through the prism. Thus, the relationship between projection distances and the system settings needs to be optimized. On the other hand, the relationship between the prism base angle and distortion as well as the match with the back-end virtual image projection system should be taken into account. As shown in Figure 4, the incident angle is preset the minimum $\theta_{1}$ and the maximum $\theta_{2}$, the minimum refraction angle $\varnothing_{1}$ and the maximum refraction angle $\varnothing_{2}$ through the prism, and the material refractive index $n$. Equation (1) is applied to calculate the required minimum prism base angle $\alpha$ :

$$
\theta_{i}=\sin ^{-1}\left(\sin \alpha \sqrt{n^{2}-\sin ^{2} \varphi_{i}}-\cos \alpha \sin \varphi_{i}\right), \quad i=1,2 .
$$

According to the parameters provided by the projector, the maximum projection emission angle appears about $34^{\circ}$, and the required minimum prism base angle about $55^{\circ}$, after the calculation. BK7 $(n=1.516)$ is used as the material for the production, and the produced prism, with the dimension $10 \mathrm{~mm} \times 7 \mathrm{~mm} \times 5 \mathrm{~mm}$, is shown in Figure 5. Using such a prism for image dividing, the horizontal angle of view of the eyebox in the laser-based pico projector is enhanced from $2.51^{\circ}$ up to $7.22^{\circ}$ that the driver could easily view the projected image. An image going through the prism has achieved the wide-view projection, while the images are not horizontally arranged. The diffraction of a holographic optical element, therefore, is needed to make the images side by side horizontally.

Then, the diffraction angle of the holographic optical element (HOE) needs to be evaluated. As the higher order of diffraction would reveal lower efficiency, the first-order diffraction is designed in this study so as to ensure the better diffraction efficiency. As shown in Figure 6, the crow-flight distance between the projection light source and the diffuser after going through the prism is assumed $s$, and the vertical distance between the projected image and the central point $h$ is assumed. In other words, the upperlayer and lower-layer images need to be vertically displaced by $h$ to parallel the middle image side by side. In this system, the distance between the projector and Image 2 is set $3 \mathrm{~cm}$, the required displacement height $h$ on the diffuser is $0.55 \mathrm{~cm}$, and the angle $\beta$ between Image 1 and Image 2 is $15^{\circ}$. The distance $d$ between the projector and Image 1 could be acquired with the trigonometric relationship, and the first-order diffraction angle is about $10^{\circ}$, according to $\theta=\tan ^{-1} d / h$. The three images could be horizontally displayed side by side through the diffraction with such an angle. In the production process, the VRP-M hologram sensitized by green light wavelength is applied to record the holographic optical element, and, according to the energy curve provided by Slavich, the hologram presents the highest diffraction efficiency at the exposure energy $75 \mu \mathrm{J} / \mathrm{cm}^{2}$, with which the required recording time is calculated [13]. Finally, the shot holographic optical element, with the length and width of $7 \mathrm{~mm}$ and $5 \mathrm{~mm}$, respectively, the grating period of $3.157 \mu \mathrm{m}$, and the diffraction angle about $9.7^{\circ}$, is shown in Figure 7.

2.2. Virtual Image Projection System. The three parallel images divided by the image-dividing element are imaged on the diffuser at the intermediate image in the virtual image projection system; a convex lens, as a projection lens, is placed at the back of the system. Before designing the virtual image projection system, the intermediate image on the diffuser is first analyzed by the resolution. The 1951 USAF resolution test chart is utilized for measuring the image resolution in this study, and the required 1951 USAF resolution test chart is established with equations (2) and (3). The result is shown in Figure 8, from which the projection limit of the projector is about the 4th element in the 1st group. The minimum resolution allowed for the projector is calculated about $0.707 \mathrm{p} / \mathrm{mm}$, which is regarded as the minimum optimization of the projection lens for the analysis: 


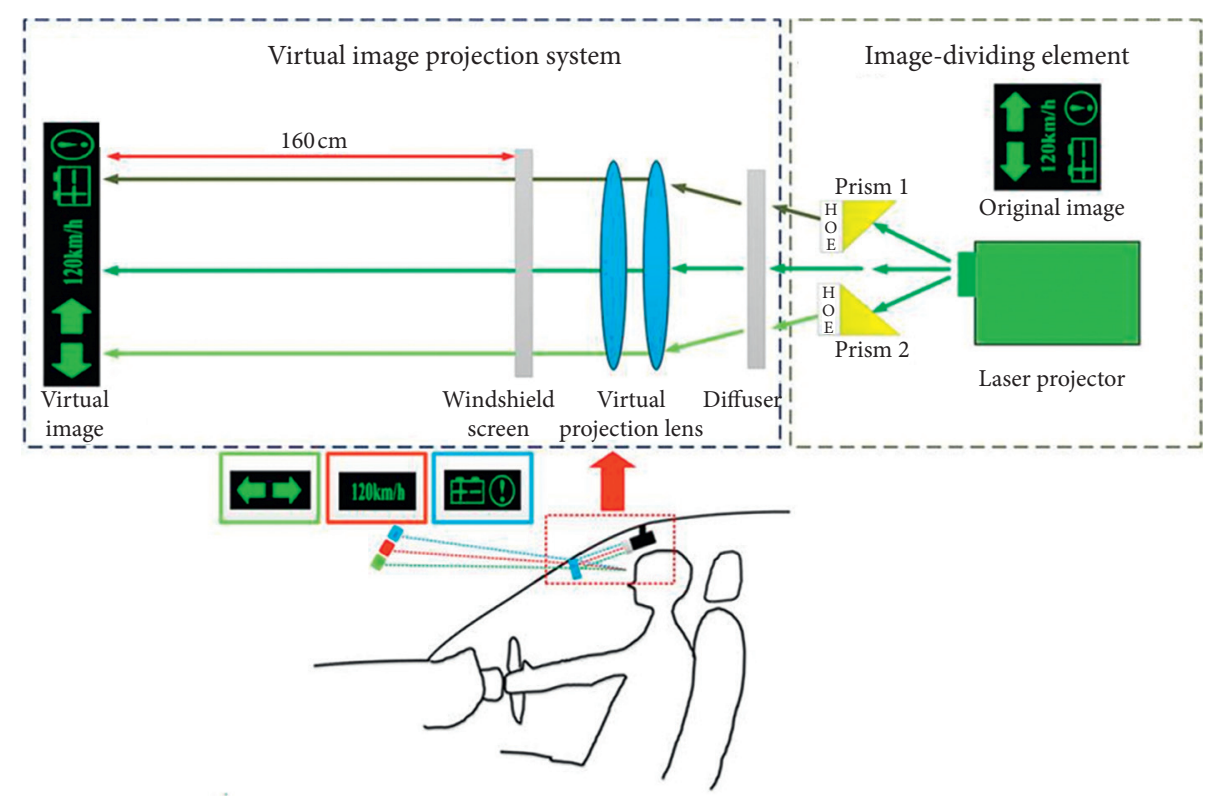

FIGURE 2: System architecture.

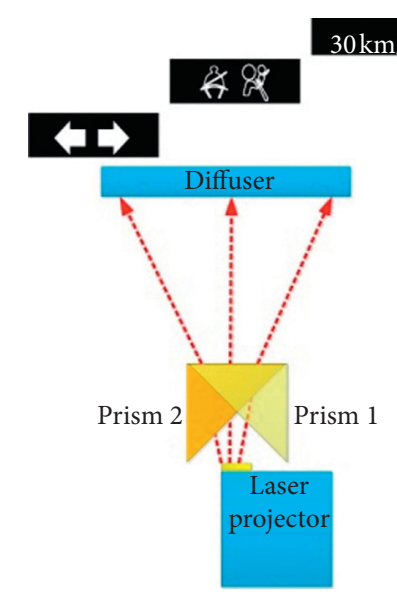

(a)

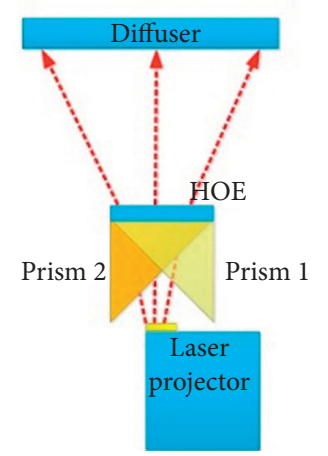

(b)

FIGURE 3: Application of the image-dividing element to the system: (a) 90-degree prisms; (b) a holographic optical element.

$$
\begin{aligned}
& \text { line length }(\mathrm{mm})=\frac{2.5 \mathrm{~mm}}{2^{\text {group }+(\text { element }-1) / 6}}, \\
& \text { line width }(\mathrm{mm})=\frac{2.5 \mathrm{~mm}}{2^{\text {group }+1+(\text { element }-1) / 6}} .
\end{aligned}
$$

In the projection system design, the object distance of the system is set $l$ and the image distance, $l^{\prime}$; the parameter of the initial order lens is calculated with the lensmaker's equation [14]. In this system, the object height is set $50 \mathrm{~mm}$ which is the imaging width of the divided image. After the design, the virtual image projection system presents the imaging at 1600 behind the combiner, the image amplification about $8.5 \mathrm{x}$, the image distortion less than $5 \%$ through the lens optimization, and the enlarged image height up to $425 \mathrm{~mm}$.

\section{Simulation}

In this study, LightTools, the nonimaging simulation software, is applied to simulate the beam splitting and Zemax, the imaging simulation software, is used for simulating the virtual image projection lens. The simulation and design is preceded with the following three steps: (1) module construction of the laser-based projector. The angle of emergence of the projector and the working principle of each pixel reflected from the laser going through the scanning lens are followed. The point light source is utilized for constructing the optical system of the laser-based projector and simulating the beam characteristics of the laser (wavelength $532 \mathrm{~nm}$ ) reflecting through MEMS; the horizontal projection angle of the system is $34^{\circ}$. (2) Design of the 


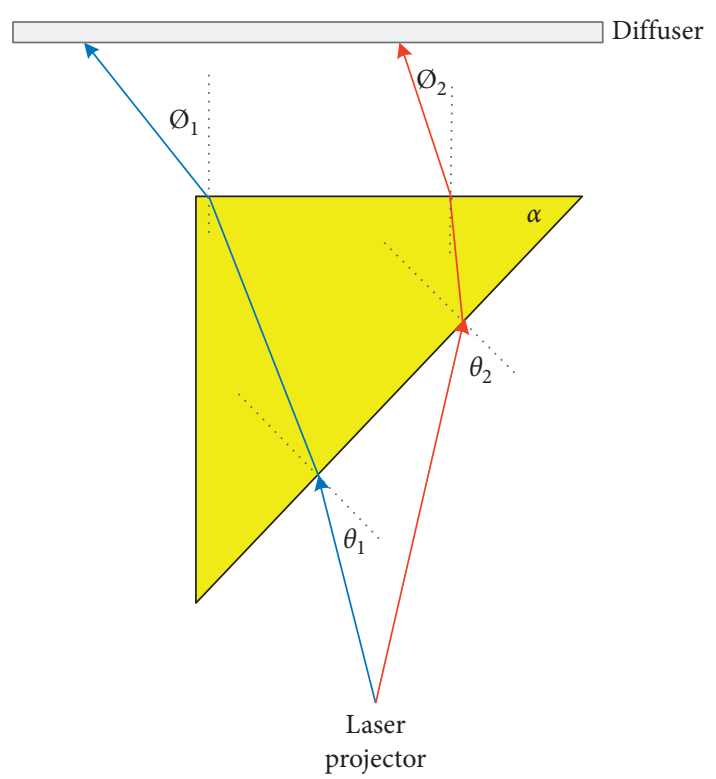

FIGURE 4: Incident angle and angle of emergence of a prism.

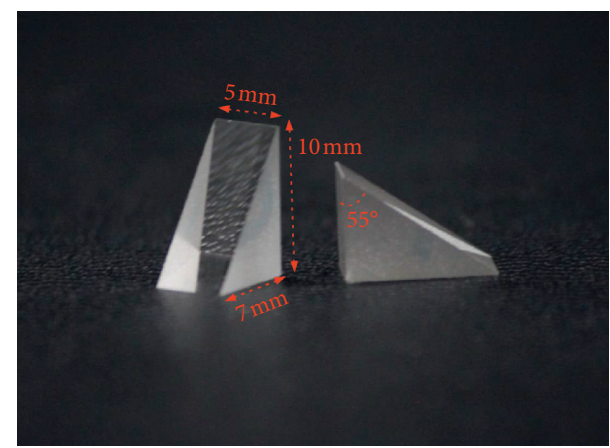

Figure 5: Photograph of the designed prisms.

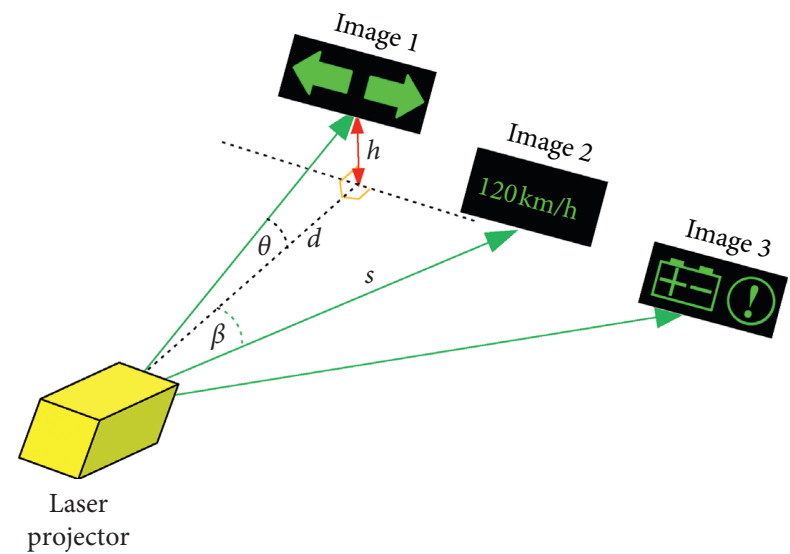

Figure 6: Evaluation of the diffraction angle.

image-dividing element, containing two 90-degree prisms and a holographic optical element. Figure 9(a) shows the simulation of optical path without adding the element. The simulation result reveals the original image size about $1 \mathrm{~cm} \times 1 \mathrm{~cm}$ being divided into 3 images through the image-dividing system, as in

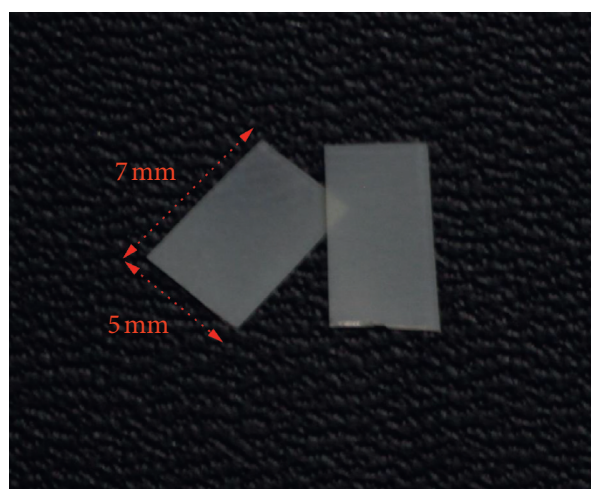

Figure 7: Photograph of designed holographic elements.

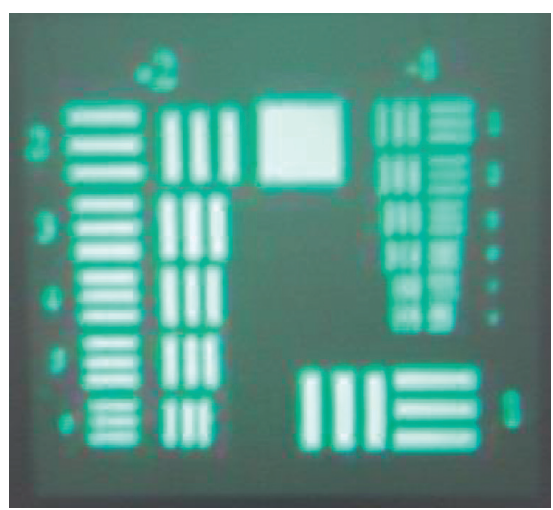

FIgURE 8: Evaluation of the 1951 USAF resolution test chart projected on the diffuser by the miniaturized projector.

Figure 9(b). From Figure 10, the horizontal projection angle of the system is about $100^{\circ}$ after the optical path being split by the beam. Such a result proves that the combination of a prism and a holographic optical element could largely increase the image width. (3) Zemax utilized for designing and evaluating the projection lens in the virtual image projection system. A projection lens is designed in this study, with the focus distance of $200 \mathrm{~mm}$ and the diffuser distancing the intermediate image of $149 \mathrm{~mm}$. The image information would be enlarged as a virtual image at $1600 \mathrm{~mm}$ behind the windshield, the lens amplification appears $8.5 \mathrm{x}$, and the virtual image sizes $27.2 \mathrm{~cm} \times 6.8 \mathrm{~cm}$. From this structure, when human eyes view the traffic image information at $45 \mathrm{~cm}$ in front of the windshield, the horizontal angle of view of the eyebox is about $7.22^{\circ}$. Figure 11 shows the relationship between field curvature of the projection structure and distortion. Figure 12 shows the Grid distortion on the virtual image projection lens. Apparently, the image distortion is controlled below $1 \%$. Human eyes generally appear about $5 \%$ sensitivity to distortion [15]. In this case, when distortion is controlled in the value, it could be accepted by human eyes.

\section{Results and Discussion}

From Figure 9, the image after the beam splitting appears some trapezium distortion because of the emission angle of the 90-degree prism and the laser projector [16]. In the real 


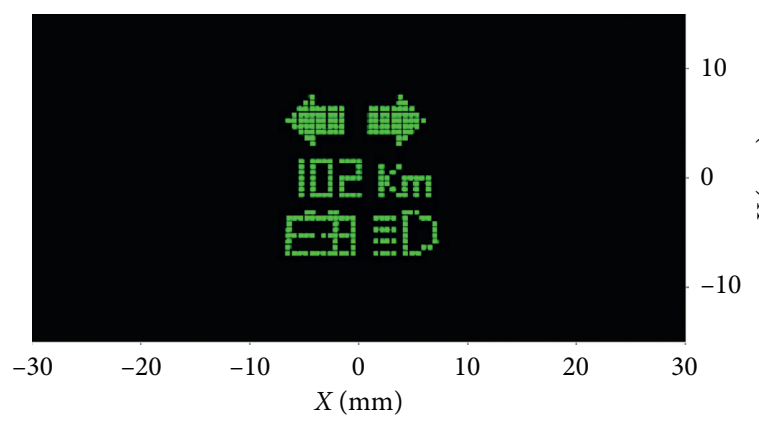

(a)

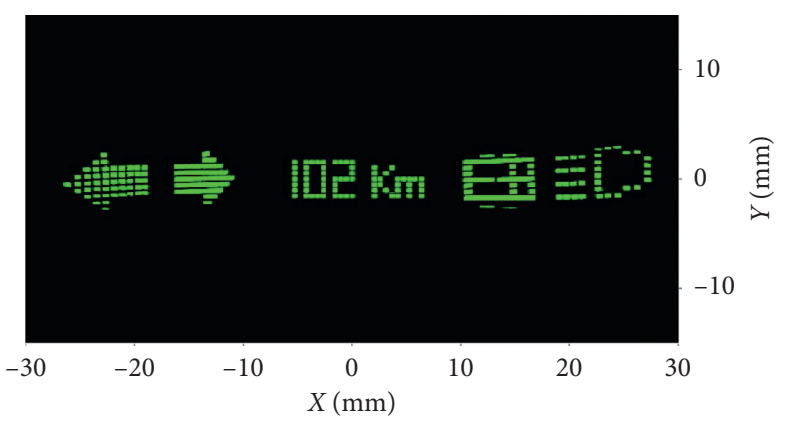

(b)

Figure 9: Imaging simulation of the image-dividing element: (a) without an element and (b) with elements.

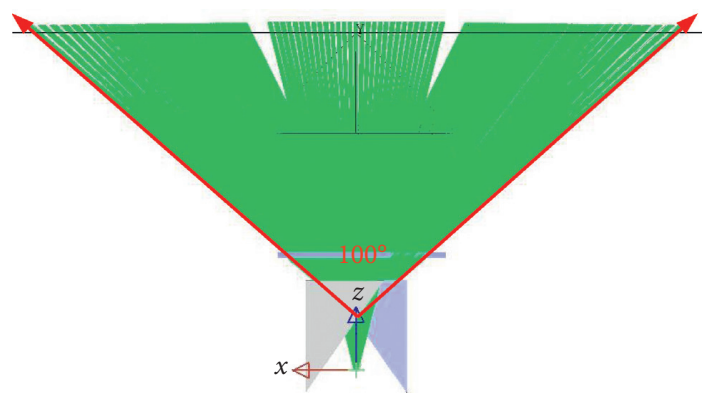

Figure 10: Optical path through the image-dividing element.
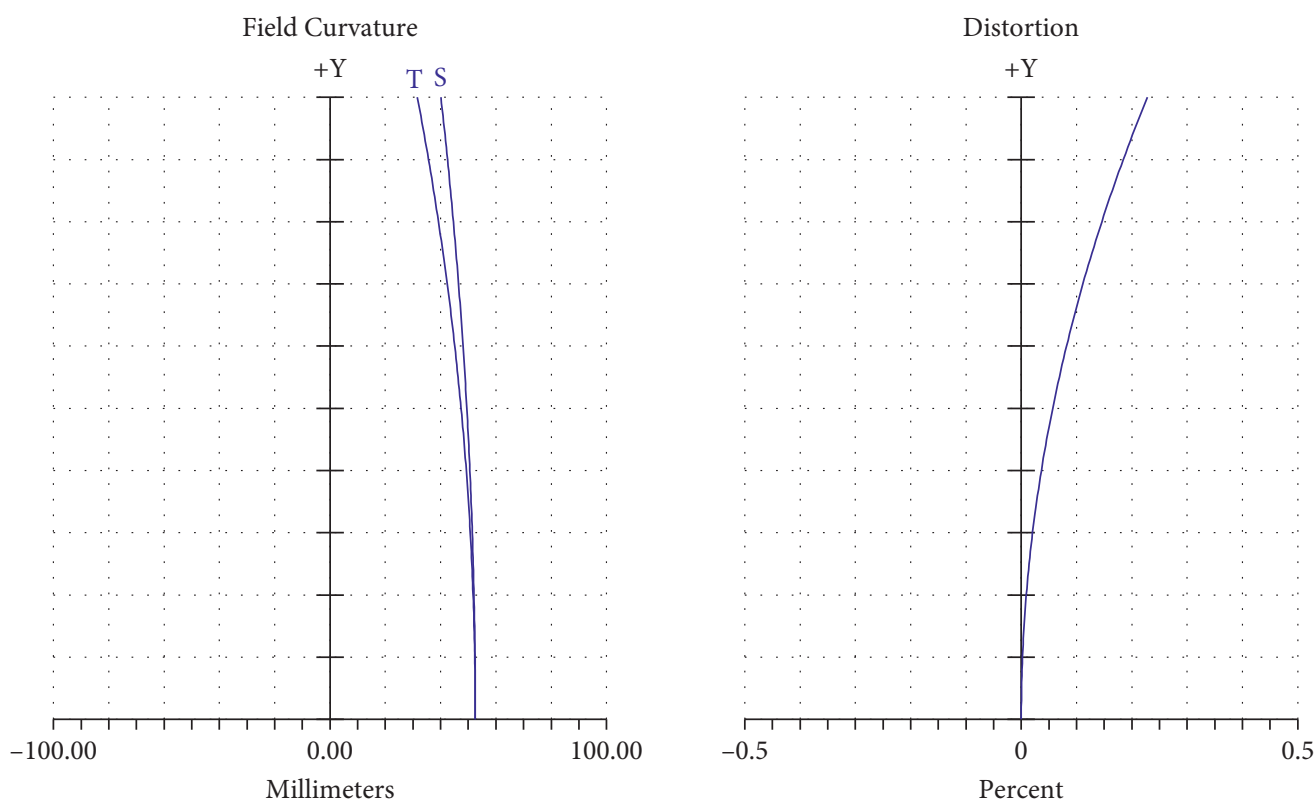

Figure 11: Field curvature and distortion of the virtual image projection lens.

system, the divided images would appear slight trapezium distortion because of the prism refraction; the original horizontal image width $1 \mathrm{~cm}$ becomes $1.15 \mathrm{~cm}$ for the upperand lower-layer image width after the deflection, showing the distortion about $+15 \%$. As a result, the horizontal width of upper- and lower-layer images needs to be reduced $15 \%$ in order to present the consistent width of the three images as well as to eliminate the distortion.
According to the simulation result, the real optical path is set up in this study, as in Figure 13. The laser-based pico projector in the simulation is regarded as an image for combining with the entire system, including the image-dividing system and the virtual image projection system, and a reflection mirror with OS-50 reflecting coating is set up in the system. $\mathrm{Ti}_{3} \mathrm{O}_{5}$ is used as the OS-50 reflecting coating material, with the reflectivity and the transmittance ratio of 


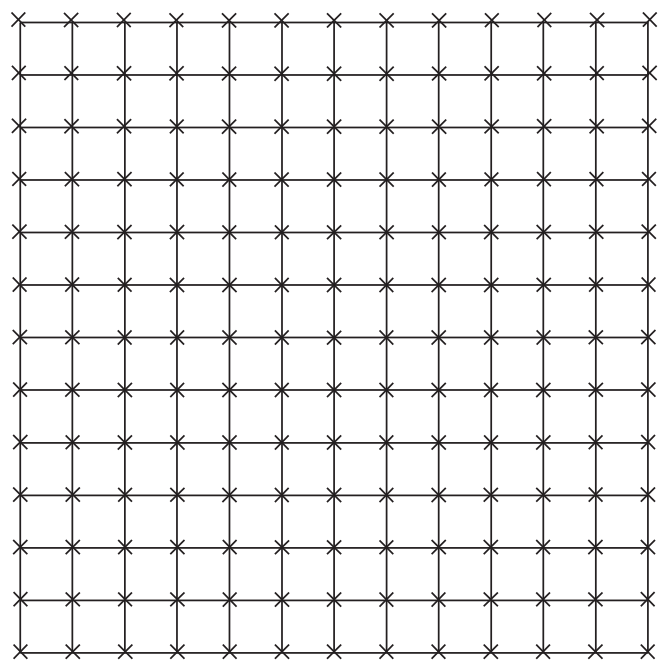

FIGURE 12: Grid distortion of the virtual image projection lens.

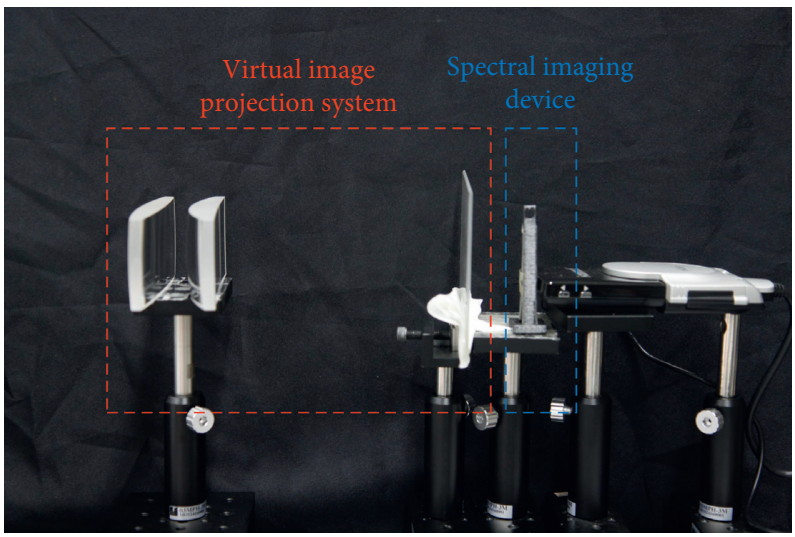

Figure 13: Photograph of the laboratory setup of the proposed system.

$4: 6$. A screen is placed at $160 \mathrm{~cm}$ behind the windshield, and a camera is used for recording the imaging. Figure 14 shows the real optical path. The projecting images are shot at daytime and in the evening.

In Figure 14, a screen is place at the image plane and a camera focuses on the image plane in order to acquire the clear image information and prove the correct imaging at $1600 \mathrm{~mm}$. The virtual image size of this system is about $26 \mathrm{~cm} \times 6.8 \mathrm{~cm}$, which is slightly smaller than the result of the simulation, with the error about 5\%. Such an error might be resulted from the $\pm 2^{\circ}$ tolerance of the prism base angle causing the deviation of the horizontal image. On the other hand, the production of a holographic optical element is comparatively complex, but the quality of chemicals or the surrounding environments could result in slight errors of holographic optical elements in the production. Such considerations have been controlled as much as possible for the consistence.

Reviewing the current head-up displays in the market, most of them directly display on a screen that the small angle of view cannot provide sufficient information for the users. Currently, a lot of research increases the image size with lenslet arrays or the amplification of projection lens $[17,18]$.
Such methods could enhance the enlargement of the image and the image height that the field of view of the driver could be obstructed. On the other hand, the three divided images can be projected by three head-up projectors, but the volume of the system might become lager and the cost might become higher [19]. In this Denso's system, four reflectors were used for dividing an image into two images and large-area overlapping technology was used for displaying the complete image. The optical components were more, and the volume of Denso's system was also large [20]. Thus, the image-dividing element produced by combining two 90-degree prisms and a holographic optical element is, therefore, proposed in this study. As a holographic optical element is light and thin, the entire system size is miniaturized, smaller than the systems with reflective elements or refractive elements, appears no distortion, and presents the fixed deflective angle; both the image quality and the installation convenience are significantly promoted.

The automobile head-up display proposed in this study could improve the projection angle of the current laser-based pico projectors from $34^{\circ}$ to $100^{\circ}$. In addition to increase the information coverage, the field of view obstruction would not 


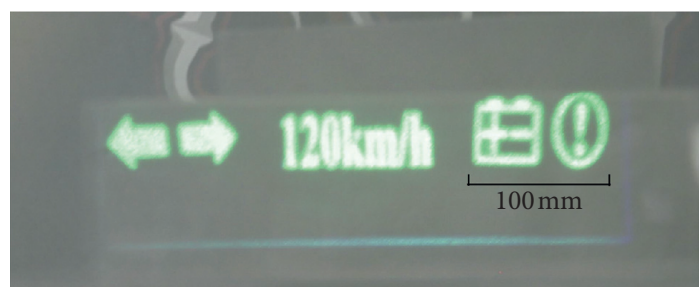

(a)

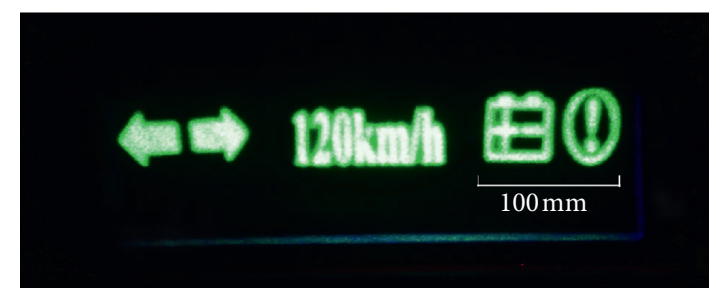

(b)

FIGURE 14: (a) Real shot simulated at daytime and (b) real shot simulated in the evening.

occur in this system. Besides, it could be combined with other types of head-up displays, providing drivers with larger horizontal field of view. Moreover, the projection distance is at $1600 \mathrm{~mm}$ behind the windshield that the augmented reality of the street scene is compatible with the projected image when a driver views the projected information. The eyes, therefore, do not need to focus on the changing view to cause eye fatigue.

\section{Conclusions}

In this study, an image-dividing element is produced by combining two 90-degree prisms and a holographic optical element and integrated with a virtual image projection system to produce a wide-view laser-based head-up display. The prism is used for horizontally deflecting the beam, and the holographic optical element is utilized for vertically deflecting the image. The images are eventually arranged as a real image horizontally on a diffuser to distinguish the left image, middle image, and right image. The divided images could be imaged at $1600 \mathrm{~mm}$ behind the windshield through the virtual image projection system. Such a virtual imaging system increases the horizontal projection angle from $34^{\circ}$ to $100^{\circ}$, the image size is enlarged into $8.5 \mathrm{x}$, and the final imaging size appears $26 \mathrm{~cm} \times 6.8 \mathrm{~cm}$. When human eyes view from $45 \mathrm{~cm}$ in front of the windshield, the horizontal angle of view of the eyebox increases from $2.51^{\circ}$ to $7.22^{\circ}$ and the size of the eyebox is $31.68 \mathrm{~cm} \times 12.48 \mathrm{~cm}$. With such a system, a driver could reduce the situation of not paying attention to or distracting from the street to view the traffic information that the accident could be relatively reduced. Meanwhile, a driver could acquire more vehicle conditions and real-time traffic conditions with the laser-based head-up display. Because the miniaturized system could reduce the space and be arranged easily in a vehicle, this proposed system could be suitable for the aftermarket.

\section{Data Availability}

The data of the optical design used to support the findings of this study are included within the article, and the data of the simulation used to support the findings of this study are available from the corresponding author upon request.

\section{Conflicts of Interest}

The authors declare that there are no conflicts of interest regarding the publication of this paper.

\section{Acknowledgments}

This research was partially supported by the Ministry of Science and Technology (MOST), Taiwan, under contract numbers MOST 107-2221-E-011-086 and MOST 107-2622E-011-007-CC3.

\section{References}

[1] M. H. Kalmanash, "Digital HUDs for tactical aircraft," in Proceedings of the Defense, Security, COCKPIT and Future Displays II, vol. 6225, Kissimmee, Florida, USA, April 2006.

[2] Y.-C. Liu, "Effects of using head-up display in automobile context on attention demand and driving performance," Displays, vol. 24, no. 4-5, pp. 157-165, 2003.

[3] R. D. Brown, D. H. Modro, and M. R. Greer, "High-resolution LCD projection based color head-up display," in Proceedings of the International Society for Optical Engineering, vol. 4362, pp. 183-193, New York, NY, USA, September 2001.

[4] M. K. Hedili, M. O. Freeman, and H. Urey, "Microlens arraybased high-gain screen design for direct projection head-up displays," Applied Optics, vol. 52, no. 6, pp. 1351-1357, 2013.

[5] M. K. Hedili, M. O. Freeman, and H. Urey, "Transmission characteristics of a bidirectional transparent screen based on reflective microlenses," Optics Express, vol. 21, no. 21, pp. 24636-24646, 2013.

[6] I.-H. Shao, W.-W. Yang, C.-H. Chen, and K.-T. Luo, "40.3: high efficiency dual mode head up display system for vehicle application," SID Symposium Digest of Technical Papers, vol. 44, no. 1, pp. 559-562, 2013.

[7] T. P. Pearsall and M. Wanninger, "LED light sources for headup displays," in Proceedings of the Photonics in the Automobile, vol. 5663, pp. 225-229, Geneva, Switzerland, 2005.

[8] C. T. Draper, C. M. Bigler, M. S. Mann, K. Sarma, and P. A. Blanche, "Holographic waveguide head-up display with 2-D pupil expansion and longitudinal image magnification," Applied Optics, vol. 58, no. 5, pp. 251-257, 2019.

[9] C. M. Bigler, M. Mann, C. Draper, A. Bablumyan, and P. A. Blanche, "Improving head-up display with waveguides and holographic optical elements," in Proceedings of the Practical Holography XXXIII: Displays, Materials, and Applications, vol. 10944, International Society for Optics and Photonics, San Francisco, CA, USA, February 2019.

[10] H. Urey, "Torsional MEMS scanner design for high-resolution display systems," in Proceedings of the Optical Scanning II, vol. 4773, pp. 27-37, Washington, DC, USA, July 2002.

[11] W.-C. Su, C.-Y. Chen, Y.-F. Wang, Y.-W. Chen, and S. S. Yang, "Effect of a diffuser on distortion reduction for a virtual image projector," Journal of Optics, vol. 13, no. 10, pp. 105401-105406, 2011.

[12] S. Sinzinger and J. Jahns, Microoptics, VCH, Vancouver, Canada, 1991. 
[13] D. Psaltis and F. Mok, "Holographic memories," Scientific American, vol. 273, no. 5, pp. 70-76, 1995.

[14] V. N. Mahajan, Optical Imaging and Aberration Part I Ray Geometrical Optics, SPIE Press, Washington, DC, USA, 1998.

[15] D. Brown, "Decentering distortion of lenses," Photogrammetric Engineering, vol. 32, pp. 362-444, 1966.

[16] X. Ning, Y. Wang, and X. Zhang, "Object shape classification and scene shape representation for three-dimensional laser scanned outdoor data," Optical Engineering, vol. 52, p. 24301, 2013.

[17] M. Sieler, S. Fischer, P. Schreiber, P. Dannberg, and A. Bräuer, "Microoptical array projectors for free-form screen applications," Optics Express, vol. 21, no. 23, pp. 28702-28709, 2013.

[18] Q.-L. Zhao, Z.-Q. Wang, Q. Sun, Z.-W. Lu, and B. O. Chen, "Simple $40^{\circ}$ head-mounted display," Optik, vol. 114, no. 4, pp. 181-183, 2003.

[19] 2012 CES, https://www.caranddriver.com/news/a18731657/ 2012-ces-audis-super-head-up-display/.

[20] N. Kanamori and Y. Hatanaka, "Head-up display apparatus," Patent US7508356, 2009. 